\title{
Globular Clusters, Dwarf Galaxies, and the Assembly of the M87 Halo
}

\author{
Eric W. Peng ${ }^{1,2}$, Hong-Xin Zhang ${ }^{3,4}$, Chengze Liu ${ }^{5}$ and Yiqing Liu ${ }^{1}$ \\ ${ }^{1}$ Department of Astronomy, Peking University, Beijing, China \\ email: peng@pku.edu.cn \\ ${ }^{2}$ Kavli Institute for Astronomy and Astrophysics, Peking University, Beijing, China \\ ${ }^{3}$ National Astronomical Observatories, Chinese Academy of Sciences, Beijing 100012, China \\ ${ }^{4}$ Departamento de Astronomía y Astrofísica, Pontificia Universidad Católica de Chile, 7820436 \\ Macul, Santiago, Chile \\ ${ }^{5}$ Center for Astronomy and Astrophysics, Department of Physics and Astronomy, Shanghai \\ Jiao Tong University, Shanghai 200240, China
}

\begin{abstract}
At the center of the nearest galaxy cluster, the Virgo cluster, lies the massive cD galaxy, M87 (NGC 4486). Using data from the Next Generation Virgo Cluster Survey, we investigate the relationship between M87, its globular clusters (GCs), and satellite dwarf galaxies. We find that the kinematics of GCs and ultra-compact dwarfs (UCDs) are different, indicating that UCDs are not simply massive GCs. We also identify a morphological sequence of envelope fraction around UCDs correlated with cluster-centric distance that suggest UCDs are the result of tidal stripping. Lastly, we find that the $[\alpha / \mathrm{Fe}]$ abundance ratios of low-mass early-type galaxies in Virgo exhibit a strong negative gradient within $\sim 400 \mathrm{kpc}$ of M87, where the galaxies closest to M87 have the highest values. These satellite galaxies are likely the surviving counterparts of accreted dwarfs that contribute stars to the metal-poor, $\alpha$-rich stellar halos of massive galaxies. Together, these results describe a dense environment that has had a strong and continuing impact on the evolution of its low-mass neighbors.
\end{abstract}

Keywords. galaxies: abundances; galaxies: evolution; galaxies: clusters : individual (Virgo); galaxies: halo; galaxies: dwarf; galaxies: individual (M87/NGC4486); galaxies: kinematics and dynamics; galaxies: stellar content

\section{Introduction}

Massive galaxies are a product of a continuous interaction with their environment. These galaxies grow by accreting gas and stars from nearby satellites. The evolution of satellite galaxies is, in turn, altered by their proximity to their host's deep gravitational potential. Low-mass galaxies, with their large numbers and their high susceptibility to environmental effects (e.g., ram pressure, tidal stripping and harassment), are some of the best targets for studying the connection between environment and the evolution of both low and high mass galaxies.

Ultra-compact dwarf galaxies (UCDs) are a particularly interesting class of object as they are thought to be the remnant nuclear star cluster of what was previously a nucleated dwarf galaxy (e.g., Bekki et al. 2003; Côté et al. 2006). If so, they are unique signposts of disrupted galaxies that have long ago donated their stars to the parent halo. Some evidence, however, has indicated that UCDs may simply be the most massive globular clusters (GCs) (Mieske et al. 2012), in which case, they may have less significance for the assembly history of stellar halos. 
To address these and other questions, we have embarked on the Next Generation Virgo Cluster Survey (NGVS, Ferrarese et al. 2012), a Large Program on the Canada-FranceHawaii Telescope (CFHT) to perform deep $u^{*} g^{\prime} i$ ' $z^{\prime}$ imaging of $104 \mathrm{deg}^{2}$ of the Virgo cluster out to the virial radii of its two main subclusters. In this contribution, we focus on the central $2^{\circ} \times 2^{\circ}$ around the $\mathrm{cD}$ galaxy, M87, a region where we have also obtained deep $K_{s}$-band photometry (Muñoz et al. 2014). One of the key advantage of NGVS imaging in the study of UCDs is that our image quality is excellent (median $i^{\prime}$-band seeing of 0.55 "). This allows us to resolve and measure sizes for objects in Virgo that are larger than $10 \mathrm{pc}$, a value typically used as a lower bound for objects considered UCDs.

\section{Kinematics of ultra-compact dwarfs around M87}

Using the NGVS imaging to identify candidate UCDs and GCs, we have carried out a systematic spectroscopic survey of compact stellar systems around M87 with the $2 \mathrm{dF} / \mathrm{AAOmega}$ multi-fiber spectrograph on the 3.9-meter AAT and the Hectospec multifiber spectrograph on the 6.5-meter MMT. Combined with data from the literature (e.g., Hanes et al. 2001; Strader et al. 2011), we analyzed a sample of 97 UCDs and 911 GCs, all spectroscopically confirmed, within $1.5 \mathrm{deg}(430 \mathrm{kpc})$ of M87 (Zhang et al. 2015). We separated UCDs from GCs based on their size $\left(r_{h}\right.$, or half-light radius) and luminosity. UCDs in our sample all have $r_{h}>11 \mathrm{pc}$ and $g^{\prime} \leqslant 21.5 \mathrm{mag}\left(M_{g} \leqslant-9.6\right)$.

Our comparison of UCD and GC kinematics revealed two significant differences. We analyzed the rotation of the UCD system compared to the systems of blue and red GCs. We found that while the blue GCs show little or no systematic rotation about M87, the UCDs have a significant rotation amplitide $(\sim 150 \mathrm{~km} / \mathrm{s})$ around an axis in-between the major and minor photometric axes. This difference suggests that the UCDs in M87 are not simply the most massive and largest GCs, for if their formation mechanism was the same, we would expect the populations to have similar kinematics.

We also inferred the orbital anisotropies of the UCDs and GCs based on the spherically symmetric Jeans equation. The anisotropy parameter, $\beta_{r} \equiv 1-\sigma_{t}^{2} / 2 \sigma_{r}^{2}$, measures the relative amount of tangential and radial velocity dispersion. We estimated the profile of $\beta_{r}$ as a function of distance from M87 for UCDs, blue GCs, and red GCs, shown in Figure 1. Once again, the blue GCs, with which UCDs are most often associated because of their similar colors, have kinematics different from the UCDs. The blue GCs have tangential orbits in both the inner and outer regions. The UCDs follow the blue GCs, having tangential orbits within $\sim 30 \mathrm{kpc}$, but then diverge from the blue GCs at larger radii, where the UCDs have predominantly radial orbits.

It is not surprising that the inner UCDs have more circular orbits, as our size-defined UCDs have at least an order of magnitude lower average density than GCs of similar luminosity, making them more vulnerable to tidal disruption at small pericenters. At large radii, however, the transition to radial orbits is suggestive of an origin that involves some tidal stripping, as would be the case if UCDs were the remnants of nucleated dwarf galaxies. Although we cannot say for certain what the origins of UCDs are, the differing kinematic profiles in the outer region between UCDs and blue GCs again suggests that they are not a single population of objects. The full description of the analysis and interpretation are in Zhang et al. (2015).

\section{A morphological sequence of ultra-compact dwarfs}

If UCDs really are the remnant nuclear star clusters of stripped galaxies, we might expect to see UCDs in different stages of being tidally stripped. Using the deep NGVS 


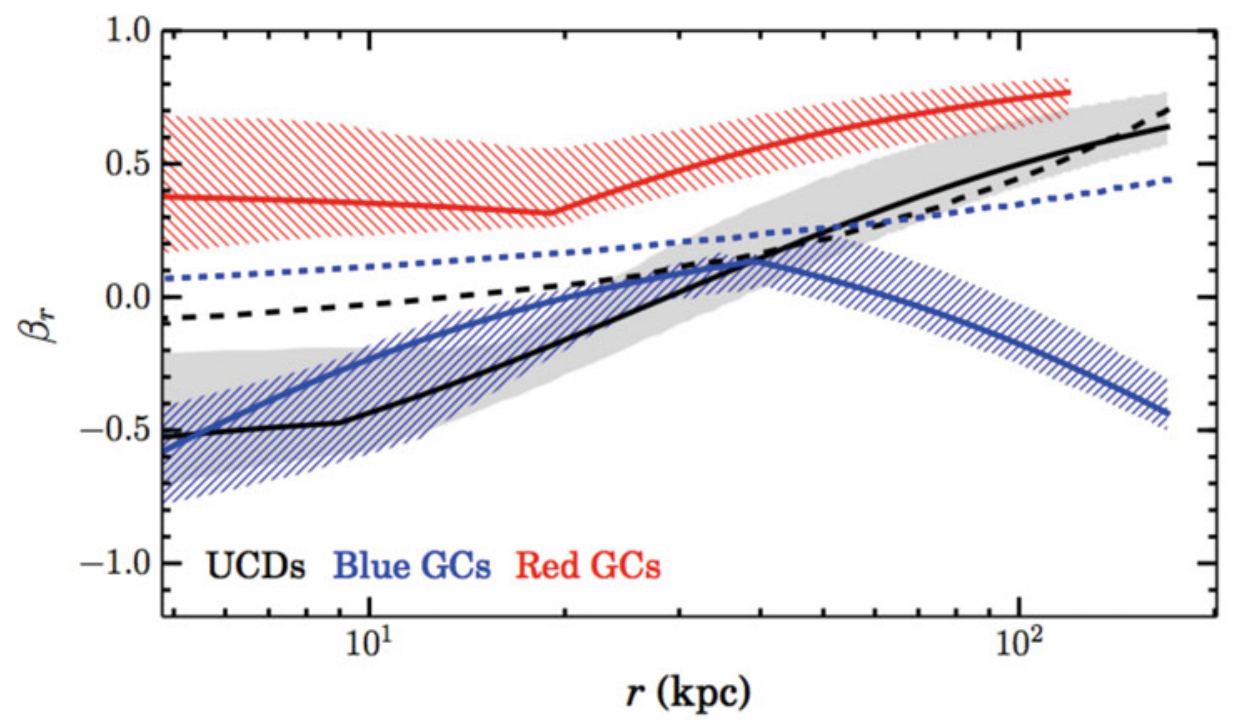

Figure 1. Variation of the orbital anisotropy as a function of the 3D radius from M87, from Zhang et al. (2015). The profiles for UCDs, blue GCs, and red GCs are represented as black, blue, and red solid curves, respectively. Following the same color code, the hatched regions of different styles mark the $68 \%$ confidence intervals for blue GCs, and red GCs. The gray shaded region marks the $68 \%$ confidence interval for the UCDs. The short dashed curves (black for UCDs, blue for blue GCs) represent the anisotropy profiles predicted by a universal relation between the number density slope and $\beta$ for relic high- $\sigma$ density peaks as found in cosmological simulations by Diemand et al. (2005).

imaging, we have performed a photometric study of the UCDs around M87 (Liu, C. et al. 2015). The NGVS $g^{\prime}$-band images reach a limiting surface brightness of 29 mag $\operatorname{arcsec}^{-2}$, giving us a new ability to detect faint envelopes around UCDs.

When we examined our UCD sample, we found that while many showed no sign of any extended envelope, a number did possess diffuse halos of varying prominence. Figure 2a shows a mosaic of nucleated, early-type dwarfs with UCDs. We find that there appears to be a continuous morphological sequence of following the visual prominence of the diffuse halo, going from a typical nucleated dwarf galaxy to a "naked" UCD.

We quantified the fraction of light contained in the envelope, and divided the UCDs into two categories, those with a visible envelope, and those with none. Figure $2 \mathrm{~b}$ shows the cumulative fraction of these two groups, as well as that of the dwarf galaxy nuclei, as a function of galactocentric distance. The UCDs without any visible envelope are the most centrally concentrated, followed by those with visible envelopes, and then by the dwarf galaxy nuclei. This result suggests that the location of the UCD or nucleus within the M87 potential well is a determining factor in the strength of any surrounding diffuse halo. Similar to the kinematic evidence described in the previous section, the radial dependence of the existence of diffuse stellar envelopes around UCDs in M87 is consistent with a tidal stripping origin for UCDs.

\section{4. $[\alpha / \mathrm{Fe}]$ in early-type Virgo dwarfs}

Even without the aforementioned example of the tidally disrupted progenitors of UCDs, it has already been well-established that low-mass galaxies contribute their stars to the stellar halos of more massive galaxies. It is also known that low-mass galaxies residing in 


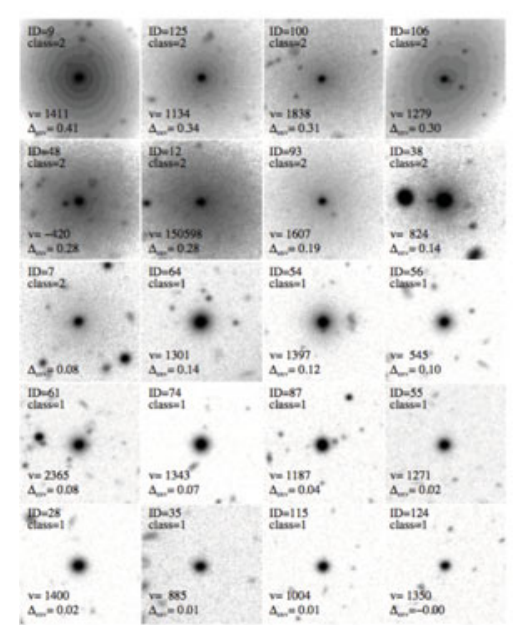

(a)

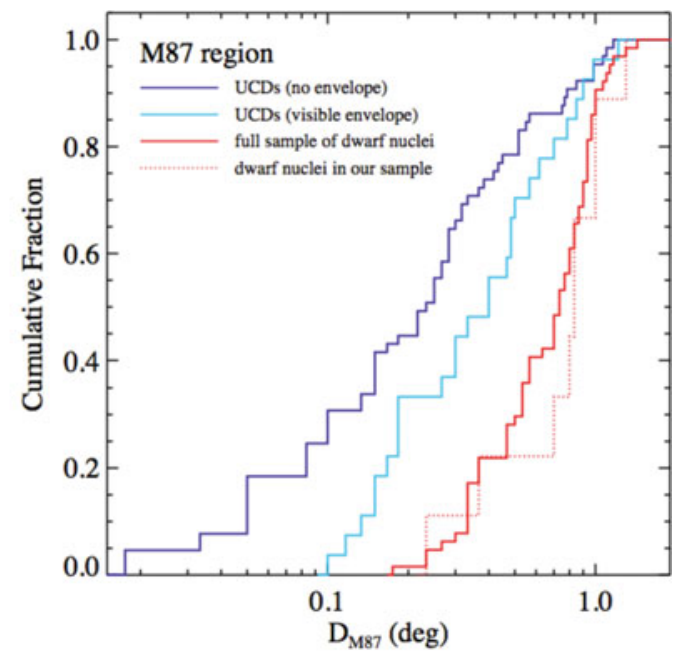

(b)

Figure 2. Left (a): Mosaic of images showing 20 objectively-selected UCD candidates around M87, from Liu, C. et al. (2015). The first nine panels show known nucleated dwarf galaxies from Binggeli et al. (1985) or new discoveries from the NGVS. The objects have been loosely arranged according to the visual prominence of an underlying diffuse halo, which is unmistakable in the first objects and non-existent in last ones. Right (b): Cumulative distributions for $D_{M 87}$ (the projected distance from M87) for UCD candidates in the M87 region (blue and cyan lines), the full sample of 64 dwarf nuclei (red solid line), and the nine additional candidates subse- quently classified as dwarf nuclei (red dotted line). The cleaned sample of 92 UCD candidates has been divided into two classes by measuring of the prominence of an outer envelope.

dense environments, or near a more massive neighbor, can have their evolution strongly altered. As a consequence, the types of stars accreted onto stellar halos are influenced by the host galaxies themselves. Greene et al. (2012, 2013) measured the mean ages, $[\mathrm{Fe} / \mathrm{H}]$, and $[\alpha / \mathrm{Fe}]$ for the outer halos of 25 massive ETGs, finding that these stellar populations had old ages, relatively low metallicity $([\mathrm{Fe} / \mathrm{H}] \sim-0.5)$, and high $[\alpha / \mathrm{Fe}] \sim$ +0.3 , a combination that had not been found in the low mass galaxies that could plausibly form an accreted population. $[\alpha / \mathrm{Fe}]$, an indicator of early star formation timescales, is typically low for low-mass galaxies, unlike the values found in the stellar halos of massive galaxies. To further explore this connection, we have investigated the timescales of star formation in low-mass Virgo early-type galaxies (ETGs) as a function of their local environment.

Peng et al. (2008) reported indirect evidence that star formation in low-mass ETGs at the center of Virgo must have proceeded rapidly, finding that galaxies closer to M87 had higher GC specific frequencies $\left(S_{N}\right)$. Assuming that massive star clusters favor intense bursts of star formation, then two galaxies with the same stellar mass but different $S_{N}$ must have different timescales over which the bulk of their stars were formed. Here, and in Liu, Y. et al. (2015), we report the results of a study of $[\alpha / \mathrm{Fe}]$ in 11 low-mass Virgo ETGs.

Figure 3 shows $[\alpha / \mathrm{Fe}]$ plotted versus projected distance from M87 for these 11 Virgo dwarf ETGs. We found a strong gradient where the galaxies closest to M87 have the highest values of $[\alpha / \mathrm{Fe}]$. This trend is only obvious, however, when looking at galaxies within $0.4 \mathrm{Mpc}$ (0.26 times the virial radius of Virgo A), all of which have super-solar $[\alpha / \mathrm{Fe}]$. Beyond this projected radius, the sample galaxies have $[\alpha / \mathrm{Fe}]$ values around 


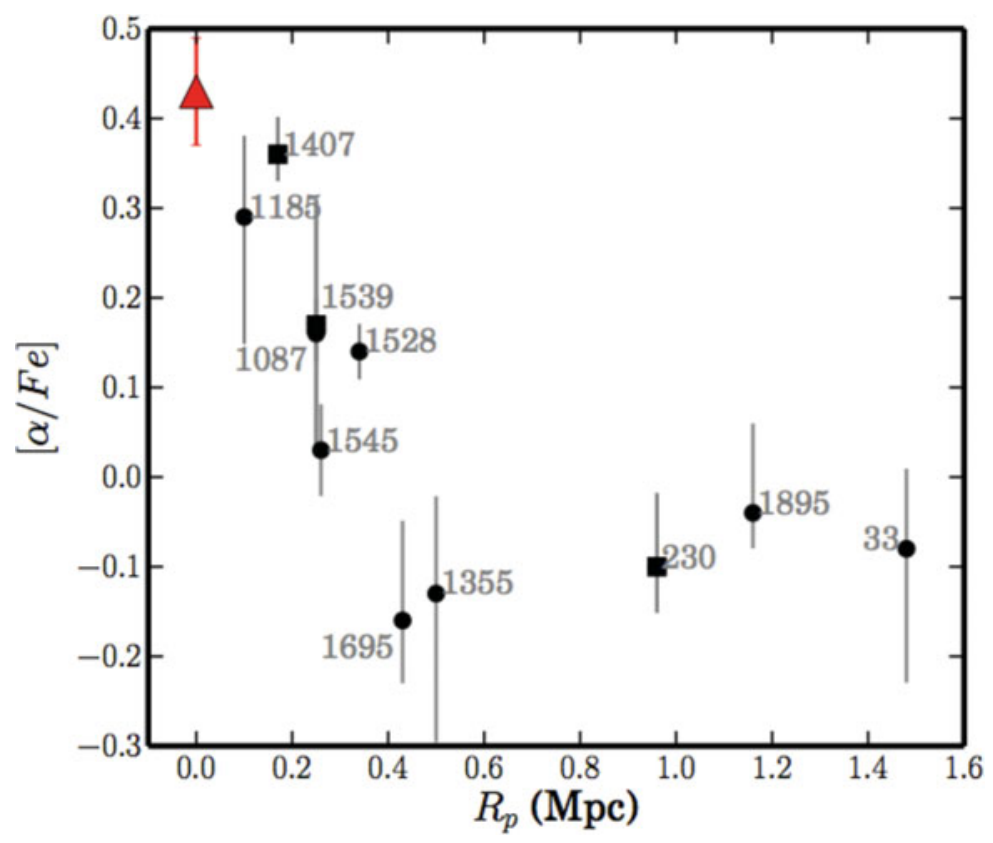

Figure 3. SSP-equivalent $[\alpha / \mathrm{Fe}]$ for low mass Virgo early-type galaxies as a function of projected distance from M87, from Liu, Y. et al. (2015). Black circles are the low mass ETGs in our sample, labeled by their VCC number. The error bars represent $68 \%$ confidence levels. $[\alpha /$ Fe] decreases with cluster-centric distance in the inner region, and then has a flat distribution in the outer regions, at values close to solar. The red triangle represents M87 within $\mathrm{R}_{e} / 2$ with 1- $\sigma$ error bar, from the work of McDermid et al. (2015).

or below solar. The innermost galaxies have $[\alpha / \mathrm{Fe}]$ values comparable to that of M87 itself suggesting that: 1) The low-mass ETGs we observe are plausible "survivors" of a parent population from which many galaxies have already accreted onto M87, and 2) Environment is the controlling factor for star formation timescales in dense regions, perhaps through quenching processes such as ram pressure stripping and strangulation.

\section{Conclusions}

In this contribution, we present results from three different investigations of the satellite population (GCs, UCDs, and dwarfs) around the Virgo cluster cD galaxy, M87. Both the kinematics and structural properties of UCDs support the idea that they are not simply massive GCs, but perhaps the remnant nuclei of tidally stripped dwarf galaxies. Our measurement of $[\alpha / \mathrm{Fe}]$ in low-mass ETGs around M87 shows that the dense environment in which the innermost galaxies reside has led to shorter star formation timescales within the central 0.4 Mpc. Taken together, these studies show that the assembly of M87's stellar halo is a result of an interplay between its deep gravitational potential and its many low-mass satellites. Future studies of the detailed chemical abundances and the internal kinematics of these satellite systems, and of the M87 halo itself, will be important for establishing the details of these links.

\section{References}

Bekki, K., Couch, W. J., Drinkwater, M. J., \& Shioya, Y. 2003, MNRAS, 344, 399

Binggeli, B., Sandage, A., \& Tammann, G. A. 1985, AJ, 90, 1681 
Côté, P., Piatek, S., Ferrarese, L., et al. 2006, ApJS, 165, 57

Ferrarese, L., Côté, P., Cuillandre, J.-C., et al. 2012, ApJS, 200, 4

Diemand, J., Madau, P., \& Moore, B. 2005, MNRAS, 364, 367

Greene, J. E., Murphy, J. D., Comerford, J. M., Gebhardt, K., \& Adams, J. J. 2012, ApJ, 750, 32

Greene, J. E., Murphy, J. D., Graves, G. J., Gunn, J. E., Raskutti, S., Comerford, J. M., \& Gebhardt, K. 2013, ApJ, 776, 64

Hanes, D. A., Côté, P., Bridges, T. J., et al. 2001, ApJ, 559, 812

Liu, C., Peng, E. W., Côté, P., et al. 2015, ApJ, 812, 34

Liu, Y., Peng, E. W., Blakeslee, J. P., et al. 2015, ApJ, submitted

McDermid, R. M., Alatalo, K., Blitz, L., et al. 2015, MNRAS, 448, 3484

Mieske, S., Hilker, M., \& Misgeld, I. 2012, A\&A, 537, A3

Muñoz, R. P., Puzia, T. H., Lançon, A., et al. 2014, ApJS, 210, 4

Peng, E. W., Jordán, A., Côté, P., et al. 2008, ApJ, 681, 197

Strader, J., Romanowsky, A. J., Brodie, J. P., et al. 2011, ApJS, 197, 33

Zhang, H.-X., Peng, E. W., Côté, P., et al. 2015, ApJ, 802, 30 